\title{
Blind ICA detection based on second-order cone programming for MC-CDMA systems
}

\author{
Chih-Wei Jen ${ }^{*}$ and Shyh-Jye Jou
}

\begin{abstract}
The multicarrier code division multiple access (MC-CDMA) technique has received considerable interest for its potential application to future wireless communication systems due to its high data rate. A common problem regarding the blind multiuser detectors used in MC-CDMA systems is that they are extremely sensitive to the complex channel environment. Besides, the perturbation of colored noise may negatively affect the performance of the system. In this paper, a new coherent detection method will be proposed, which utilizes the modified fast independent component analysis (FastICA) algorithm, based on approximate negentropy maximization that is subject to the second-order cone programming (SOCP) constraint. The aim of the proposed coherent detection is to provide robustness against small-to-medium channel estimation mismatch (CEM) that may arise from channel frequency response estimation error in the MC-CDMA system, which is modulated by downlink binary phase-shift keying (BPSK) under colored noise. Noncoherent demodulation schemes are preferable to coherent demodulation schemes, as the latter are difficult to implement over time-varying fading channels. Differential phase-shift keying (DPSK) is therefore the natural choice for an alternative modulation scheme. Furthermore, the new blind differential SOCP-based ICA (SOCP-ICA) detection without channel estimation and compensation will be proposed to combat Doppler spread caused by time-varying fading channels in the DPSK-modulated MC-CDMA system under colored noise. In this paper, numerical simulations are used to illustrate the robustness of the proposed blind coherent SOCP-ICA detector against small-to-medium CEM and to emphasize the advantage of the blind differential SOCP-ICA detector in overcoming Doppler spread.
\end{abstract}

Keywords: Multicarrier code division multiple access (MC-CDMA); Second-order cone programming (SOCP); Independent component analysis (ICA); Negentropy

\section{Introduction}

The use of multicarrier code division multiple access (MC-CDMA) has recently been proposed for future wireless communication systems, and cellular networks in particular [1], due to its high data rate or spectrum efficiency over frequency-selective fading channels [2,3]. MC-CDMA combines orthogonal frequency-division multiplexing (OFDM) with CDMA to spread the data symbol in the frequency domain. The main advantage of OFDM is that the narrowband of the subcarriers may be significantly increased in order to combat frequency-selective fading. As opposed to the spreading of data in the time domain, as in the direct sequence CDMA technique, the spreading of data associated with the subcarriers in MC-

\footnotetext{
* Correspondence: charlesjen10@gmail.com

Department of Electronics Engineering, National Chiao Tung University, Hsinchu 30010, Taiwan
}

CDMA is carried out in the frequency domain. In order to ensure orthogonality among users in the frequency domain, Walsh-Hadamard sequences are often implemented to achieve the spreading [4]. However, a disadvantage of the MC-CDMA system is that it is easily affected by imperfect channel frequency response estimation mismatch [5]. Besides, the system performance may suffer from Doppler spread, which is induced by time-varying fading channels [6].

In general, coherent receivers that utilize channel estimation and compensation achieve a gain of approximately 3 to $4 \mathrm{~dB}$ in signal-to-noise ratio (SNR) compared to noncoherent receivers [7] under additive white Gaussian noise (AWGN). Therefore, the design of coherent receivers is based on the assumption that the channel estimate is error-free. However, coherent demodulation is difficult to achieve over time-varying fading channels, as this requires

\section{实}


frequency and phase synchronization of all carriers, and therefore, it is logical to consider differential phase-shift keying (DPSK) as an alternative modulation scheme [8].

It should also be mentioned that the performance of the system at the receiver end will be degraded by the perturbation of colored noise, which can be defined as the variation in power spectral density in the frequency domain, and may be induced by narrowband interference (NBI) $[9,10]$, intercarrier interference (ICI) in wideband channels [10,11], oversampling [12], channel shortening [13], analog bandpass filter [14], etc. in multicarrier systems. In most practical circumstances, white noise is simply a particular instance of colored noise. The assumption of the presence of colored noise is therefore often much more realistic than assuming white noise, which may render difficulties in implementation.

A large number of blind multiuser detection methods, making use of only the spreading sequence and timing of the desired user, have been proposed. The main reasoning behind employing a blind detector is to avoid the requirements of a training sequence and the reduced loss of channel throughput. Suboptimal approaches to the use of blind multiuser detectors, such as $[15,16]$, have also been proposed to mitigate multiple access interference (MAI), which results from multipath fading destroying the orthogonality of the user's spreading sequences. The performance of each of these methods is heavily dependent on accurate knowledge of the channel impulse response or the signature waveform of the user of interest at the receiver end. Unfortunately, in most real-world, practical circumstances, a perfect estimation of the information within channel parameters cannot be conveyed to the receiver, resulting in the system's performance degradation accordingly [17-20]. In [15], Honig et al. propose a blind version of the linear minimum mean square error (LMMSE) detector, using constrained minimum output energy (CMOE) as the cost function for MAI suppression. However, in this method, the receiver's performance is considerably degraded in the presence of channel and data correlation matrix estimation errors [21,22].

There has been substantial research into the use of subspace-based blind receivers $[18,23]$ in the MC-CDMA system. The second-order statistic (SOS)-based technique utilizes eigenvalue decomposition (EVD) on the covariance matrix of the received signal. However, in this technique, the orthogonality between the signal and noise subspaces is subject to serious destruction due to correlated noise subspace $[24,25]$. Another effective diagonal loading technique [26] has been the widely used approach of enhancing robustness against mismatch errors and correlated noise. The main drawback of this method is that there is uncertainty as to how to precisely choose the optimal value of the diagonal loading factor [27]. In [28], Wang et al. utilize a blind variable- diagonal loading (VDL) multiuser detector, based on the concept of optimizing the worst-case performance over properly chosen bounded uncertainty sets that are determined by a bounding probability, to provide further robustness against small-to-medium prior signal modeling errors in MC-CDMA systems.

Another extensively studied technique is the blind separation of sources (BSS) based on higher-order statistics (HOS), which has a potential for application in a variety of areas, such as biomedical engineering, sonar, radar, speech enhancement, and telecommunications [29-32]. SOS-based techniques usually require the power spectra of the sources to be different [24], while in general, HOSbased techniques depend on the non-Gaussian sources in the probability distribution, even if the sources have the same power spectra. The HOS-based independent component analysis (ICA) algorithm has been implemented to deal with BSS of unknown sources from a mixed-up received signal. This algorithm is based on the assumption of the independence and non-Gaussianity of the sources before maximizing the approximate negentropies of those ones [30,33]. The approximate negentropy can be described by choosing a proper nonlinear cost function. Thereafter, the classical ICA algorithm performs the pre-processing steps, which include centering and whitening $[33,34]$ based on eigenvector projection, to decorrelate the mixed-up received signal, following which it separates the mixture by using a 'Gram-Schmidt-like' technique [30,33] after every iteration step under unit variance constraint. However, when using this technique, the weight order of the source separation will be unpredictable, a phenomenon which is referred to as weight order ambiguity $[30,35]$.

The application of ICA has been extended to dealing with complex-valued sources, in addition to real-valued sources [36-40]. In [39], two types of receivers, the RAKEICA and MMSE-ICA detectors, have been proposed, which are developed by modifying the classical complexvalued fast independent component analysis (FastICA) algorithm [36], based on the quasi-Newton iteration as well as the proper starting point initializing iterations. Furthermore, a modified stochastic gradient descent ICA detector [40] has been generalized from Amari's natural gradient algorithm [41]. All of these ICA-based detectors first carry out the centering and whitening steps and then select the appropriate starting point initializing iterations for capturing the correct weight vector. If colored noise exists, the whitening based on eigenvector projection is unable to decorrelate the mixed-up signals [42,43], which means that the system's performance is degraded accordingly.

Under colored noise environment, the SOS-based technique will fail since some of the correlated noise subspace diverges into the signal eigenvectors causing serious destruction to this orthogonality especially at low SNR [25]. The HOS-based ICA algorithm depends on the non- 
Gaussian sources in the probability distribution for BSS and feature extraction [30,33]. Available information may be used to solve the problem of weight order ambiguity, by either indirectly choosing appropriate close point initializing ICA iterations [44] or directly adding the additional constraint [42,45]. Besides, the signal processing technique proposed in $[46,47]$ utilizes the worst-case performance optimization based on second-order cone programming $(\mathrm{SOCP})$ constraint to mitigate estimation error. Therefore, in this paper, a new blind coherent detection will be developed, which utilizes the modified FastICA algorithm [36] and the optimization of the lower bound of the worst-case performance, based on approximate negentropy maximization, as well as the concept of SOCP constraint $[46,47]$. This method initializes iterations using appropriate starting points to improve robustness against small-to-medium channel frequency response estimation mismatch in the downlink binary phase-shift keying (BPSK)-modulated MC-CDMA system under colored noise.

The application will then be extended to combat large channel estimation mismatch (CEM); that is, the design will be associated with DPSK demodulation without channel estimation and compensation in order to overcome Doppler spread caused by time-varying fading channels in the noncoherent MC-CDMA system under colored noise.

The coherent and differential SOCP-ICA detectors proposed in this paper are capable of dealing with the weight order ambiguity present in the classical ICA algorithm $[33,36]$, converging on the correct solution over complex channels with colored noise. Computer simulations indicate that the performance of the proposed blind multiuser detectors is superior to those in existing literature.

The remaining of this paper is organized as follows. 'Section 2' describes the signal model for the downlink MC-CDMA system over Rayleigh fading channel. 'Section 3' respectively proposes the blind coherent and differential SOCP-ICA detectors to combat against the small-tomedium CEM and Doppler spread under colored noise. 'Section 4' compares both computational complexities with other methods. Simulation results are shown and discussed in 'Section 5', and 'Section 6' concludes this paper.

\section{Signal model}

Consider a synchronous downlink MC-CDMA system with $M$ orthogonal subcarriers and $K$ active users. First, the $i$ th input data symbol of the $k$ th user, $b_{k}(i) \in\{1,-1\}$ $(k \in\{1,2, \cdots, K\})$, is replicated $M$ times; each of these $M$ parallel copies is spreaded by the corresponding chip of the kth user's orthogonal Walsh-Hadamard code, $\quad c_{k}(m)_{m=0,1, \cdots, M-1} \in\{1 / \sqrt{M},-1 / \sqrt{M}\}$, in the frequency domain. Then, the spreaded symbol is sent to an $M$-point inverse discrete Fourier transform (IDFT) to convert the spread data into the time domain. After the parallel-to-series conversion, a cyclic prefix $(\mathrm{CP})$ - presumed to be longer than the maximum delay spread - is inserted to eliminate intersymbol interference (ISI) caused by multipath fading. For the user $k$, the downlink symbols are finally transmitted over the frequency-selective Rayleigh fading channels and their equivalent effect in discrete time is a finite impulse response tap-delay line filter with channel impulse response $\beta_{k}(0), \beta_{k}(1), \cdots, \beta_{k}$ $\left(L_{k}-1\right)$, where $L_{k}$ is the channel order of the $k$ th user. It is assumed that the narrowband signal at each subcarrier suffers from flat fading.

At the receiver end, after removing the $\mathrm{CP}$, the resultant is then passed through a series-to-parallel conversion and sent to an $M$-point DFT to convert the data into the frequency domain. For the desired user 1, the received baseband MC-CDMA signal in the frequency domain can be represented as

$$
\mathbf{y}(i)=\sum_{k=1}^{K} \mathbf{Q}_{1} \sqrt{P_{k}} \mathbf{c}_{k} b_{k}(i)+\mathbf{n}(i)
$$

where $\sqrt{P_{k}}$ is the chip energy of the $k$ th user, $\mathbf{c}_{k}=\left[c_{k}(0)\right.$, $\left.c_{k}(1), \cdots, c_{k}(M-1)\right]$ is the normalized spreading code vector of the $k$ th user, and $\mathbf{Q}_{1}=\operatorname{diag}\left\{q_{1,0}, q_{1,1}, \ldots, q_{1, M-1}\right\}$ denotes the channel matrix of the first user, whose diagonal elements are the channel frequency response given by $q_{1, m}=\sum_{l=0}^{L_{1}-1} \beta_{1}(l) e^{-j 2 \pi m l / M}, m=0,1, \cdots, M-1$. It is assumed that $\mathbf{n}(i)=\left[n_{i}(0), n_{i}(1), \ldots, n_{i}(M-1)\right]^{T}$ is a colored noise vector, each element of which contains an additive channel noise that is a zero-mean random variable with a variance of $\sigma_{n}^{2}$.

\section{Blind ICA detection based on SOCP constraint}

The HOS-based ICA algorithm is actually a neural network learning rule transformed into an efficient fixed-point iteration. It is based on an assumption of the independence and non-Gaussianity of the sources. Due to using the CDMA technique, it is rationally presumed that each user and path is approximately independent of each other, so each received data sequence can be regarded as an independent source sequence. Afterward, specific features of the received mixed-up signals will be extracted by maximizing their approximate negentropies.

First, the differential entropy $H$ of a continuous random vector $\mathbf{z}=\left[z_{1}, z_{2}, \cdots, z_{K}\right]^{T}$ with density $f(\mathbf{z})$ is defined as $H(\mathbf{z})=-\int f(\mathbf{z}) \log f(\mathbf{z}) d \mathbf{z}$ [48]. The $k$ th random variable $z_{k}$ is assumed to be of zero mean and unit variance for $k=0,1, \cdots, K$. Negentropy can be simply obtained from differential entropy and defined $[30,33]$ as

$$
J(\mathbf{z})=H\left(\mathbf{z}_{\text {Gauss }}\right)-H(\mathbf{z}),
$$


where $\mathbf{z}_{\text {Gauss }}$ is a Gaussian random vector of the same covariance matrix as $\mathbf{z}$. Mutual information is the theoretical measure of the dependence between random variables. Then, exploiting negentropy, mutual information can be expressed $[30,33]$ as

$$
I\left(z_{1}, z_{2}, \cdots, z_{K}\right)=J(\mathbf{z})-\sum_{k=1}^{K} J\left(z_{k}\right),
$$

where $J(\mathbf{z})$ is a constant for a given covariance matrix. Maximizing the sum of the negentropy of the components subject to a variance constraint equals minimizing mutual information while estimating independence. Therefore, it is clearly shown from (3) that minimizing the mutual information is roughly equivalent to finding the direction in which the negentropy is maximized. Negentropy is a measure that is zero for a Gaussian variable and always nonnegative. An approximation of negentropy for the $k$ th signal can be given [33] by

$$
J\left(z_{k}\right) \approx \xi\left[E\left\{G\left(z_{k}\right)\right\}-E\{G(v)\}\right]^{2},
$$

where $E\{\cdot\}, G$, and $\xi$ denote, respectively, the expectation, any nonquadratic function, and an irrelevant constant and $v$ is a Gaussian variable of zero mean and unit variance. The random variable $z_{k}$ is assumed to be of zero mean and unit variance [30,33]. The approximation of negentropy given in (4) readily provides a cost function for carrying out the ICA. It can measure the non-Gaussianity of data sequences by maximizing their approximate negentropies. Generally speaking, super-Gaussian cases, including binary sources, are extensively used and result in $E\left\{G\left(z_{k}\right)\right\}>E\{G(v)\}[49]$ with respect to a proper $G$ function. Therefore, the maximum value of (4) is obtained at a certain optimum of $E\left\{G\left(z_{k}\right)\right\}$ [33] that is reduced to maximizing $E\left\{G\left(z_{k}\right)\right\}[36,49]$. Maximizing the negentropy could be interpreted as an estimation of a single independent component, which means that if $J\left(z_{1}\right)$ is the largest negentropy among all $J\left(z_{k}\right), z_{1}$ has the largest non-Gaussianity.

For complex-valued sources, find a certain signal tap and make $z_{k}=\mathbf{w}^{H} \mathbf{y}$, where $\mathbf{w}$ is a certain signal tap-weight vector and the superscript $H$ denotes the complex conjugate transposition. This inference is extended to complexvalued sources in $[36-38,49]$, making the cost function

$$
J\left(z_{k}\right)=E\left\{G\left(\left|\mathbf{w}^{H} \mathbf{y}\right|^{2}\right)\right\} .
$$

Then, maximizing (5) subject to unit variance constraint $E\left\{\left|\mathbf{w}^{H} \mathbf{y}\right|^{2}\right\}=1$, the weight vector $\mathbf{w}$ of the classical complexvalued FastICA can obtain the optimization problem [36]

$$
\underset{\mathbf{w}}{\operatorname{maximize}} E\left\{G\left(\left|\mathbf{w}^{H} \mathbf{y}\right|^{2}\right)\right\}, \text { subject to } E\left\{\left|\mathbf{w}^{H} \mathbf{y}\right|^{2}\right\}=1 \text {. }
$$

It has been demonstrated through stability analysis [33] that almost any nonquadratic, even function can be regarded as a cost function for non-Gaussianity maximization. For super-Gaussian signals, a suitable choice of the $G$ function is a smooth, even function with fast computation, such as $G=\log \left(0.1+\left|\mathbf{w}^{H} \mathbf{y}\right|^{2}\right)$. Independent components with super-Gaussian distribution tend to be found first $[33,36]$.

To achieve distinct convergent points, a Gram-Schmidtlike orthogonalization technique [33] is generally applied after every ICA iteration step to separate the signals of each user. However, the weight order of the source separation is unpredictable [30]. Even though the desired weight will be captured using centering and pre-whitening [33,34], based on eigenvector projection and an appropriate starting point initializing ICA iterations [39], it is still sensitive and subject to the complex channel model.

Available information may be used to solve the problem of weight order ambiguity, either by indirectly choosing appropriate close point initializing ICA iterations [44] or by directly adding the additional constraint $[42,45]$. ICA is a higher-order, statistical signal processing technique for BSS and features extraction by maximizing the approximate negentropy of input signals, which is significantly less sensitive to the colored noise model than the SOSbased EVD [24]. Therefore, the proposed idea - which imposes the additional SOCP constraint in the classical, complex-valued FastICA algorithm and then initializes iterations using the desired spreading code - could attain an adaptive, interference suppression technique for coherent and differential detection over complex channels with colored noise.

\subsection{Robust coherent SOCP-ICA detection}

In the coherent BPSK-modulated MC-CDMA system, the received data vector $\hat{\mathbf{y}}(i)$ at the desired receiver end has been implemented by channel estimation and compensation and can be represented as

$$
\begin{aligned}
\hat{\mathbf{y}}(i) & =\hat{\mathbf{Q}}_{1}^{-1} \mathbf{y}(i) \\
& =\sqrt{P_{1}} \hat{\mathbf{c}}_{1} b_{1}(i)+\mathbf{s}_{\mathrm{int}}+\hat{\mathbf{Q}}_{1}^{-1} \mathbf{n}(i),
\end{aligned}
$$

where $\hat{\mathbf{Q}}_{1}$ is the estimated channel frequency response matrix of the desired user, $\hat{\mathbf{c}}_{1}=\hat{\mathbf{Q}}_{1}^{-1} \mathbf{Q}_{1} \mathbf{c}_{1}$ is defined as the presumed (i.e., compensated) spreading signature, and $\mathbf{s}_{\text {int }}=\sum_{k=2}^{K} \hat{\mathbf{Q}}_{1}^{-1} \mathbf{Q}_{1} \sqrt{P_{k}} \mathbf{c}_{k} b_{k}(i)$ is the interference vector with regard to user 1 . It is usually assumed that the presumed spreading signature $\hat{\mathbf{c}}_{1}$ has perfect knowledge of its spreading code vector, $\hat{\mathbf{c}}_{1}=\mathbf{c}_{1}$, but this may not be true in real systems since the presumed spreading signature $\left(\hat{\mathbf{c}}_{1}\right.$ equaling $\mathbf{c}_{1}$ ) may include CEM and is therefore nominal. Under unknown CEM, consider the error between the 
actual desired spreading code vector $\mathbf{c}_{1}$ and its presumed value $\hat{\mathbf{c}}_{1}$,

$$
\hat{\mathbf{c}}_{1}=\mathbf{c}_{1}+\Delta \mathbf{e},
$$

where $\Delta \mathbf{e}$ denotes an unknown, complex mismatch vector of the desired user. Since the channel frequency response estimation error is known to be complex, circularly symmetric, and Gaussian-distributed [5], it is reasonably inferred that the mismatch term $\Delta \mathbf{e}$ is modeled as a vector of complex, circularly symmetric, Gaussian random variables with zero mean and a variance of $\sigma_{e}^{2}$.

Now, it is assumed that the norm of mismatch vector $\Delta \mathbf{e}$ would be bounded by a small-to-medium value $\delta$, i.e., $\|\Delta \mathbf{e}\| \leq \delta$, where $\|\cdot\|$ denotes the Frobenius norm of a vector. The presumed spreading signature $\hat{\mathbf{c}}_{1}$ is expressed as a vector in the set

$$
C_{1}(\delta)=\left\{\hat{\mathbf{c}}_{1} \mid \hat{\mathbf{c}}_{1}=\mathbf{c}_{1}+\Delta \mathbf{e},\|\Delta \mathbf{e}\| \leq \delta\right\} .
$$

For constraint satisfaction, the weight vector $\mathbf{w}$ must satisfy $\left|\mathbf{w}^{H} \hat{\mathbf{c}}_{1}\right| \geq 1$ for all vectors $\hat{\mathbf{c}}_{1} \in C_{1}(\delta)$, where $\mathbf{w}$ is the desired signal tap-weight vector of the multiuser detector. Such a constraint guarantees that the data symbols of user 1 can be taken from the mixture, regardless of how its channel estimation is mismatched, while the error is bounded by $\delta$. Now suppose that the weight constraint is enforced; the main goal will be to find a vector $\mathbf{w}$ that maximizes the cost function $E\left\{G\left(\left|\mathbf{w}^{H} \hat{\mathbf{y}}\right|^{2}\right)\right\}$. Then, the problem of the worst-case design of the ICA detector based on approximate negentropy maximization can be formulated as

$$
\begin{array}{cc}
\underset{\mathbf{w}}{\operatorname{maximize}} & E\left\{G\left(\left|\mathbf{w}^{H} \hat{\mathbf{y}}\right|^{2}\right)\right\}, \text { subject to }\left|\mathbf{w}^{H} \hat{\mathbf{c}}_{1}\right| \geq 1 \\
& \text { for }\|\Delta \mathbf{e}\| \leq \delta .
\end{array}
$$

The problem in (10) can be rewritten as

$$
\underset{\mathbf{w}}{\operatorname{maximize}} E\left\{G\left(\left|\mathbf{w}^{H} \hat{\mathbf{y}}\right|^{2}\right)\right\} \text {, subject to } \min _{\|\Delta \mathbf{e}\| \leq \delta}\left|\mathbf{w}^{H} \hat{\mathbf{c}}_{1}\right| \geq 1 .
$$

The ICA formulation introduces the additional SOCP condition [46], which has infinitely many nonconvex quadratic constraints on $\mathbf{w}$. According to the derivation of [50], it can be easily shown that in the case of a sufficiently small uncertainty (i.e., where the uncertainty radius $\left.\delta \leq\left|\mathbf{w}^{H} \mathbf{c}_{1}\right| /\|\mathbf{w}\|\right)$, the left-hand side of the constraint in (11) can be rewritten as

$$
\min _{\|\Delta \mathbf{e}\| \leq \delta}\left|\mathbf{w}^{H}\left(\mathbf{c}_{1}+\Delta \mathbf{e}\right)\right|=\left|\mathbf{w}^{H} \mathbf{c}_{1}\right|-\delta\|\mathbf{w}\| .
$$

Using (12) and taking into account that the cost function in (10) is not affected by an arbitrary phase rotation of $\mathbf{w}$, the problem of (11) can be described in this form:

$$
\underset{\mathbf{w}}{\operatorname{maximize}} E\left\{G\left(\left|\mathbf{w}^{H} \hat{\mathbf{y}}\right|^{2}\right)\right\}, \quad \text { subject to } \mathbf{w}^{H} \mathbf{c}_{1}-\delta\|\mathbf{w}\| \geq 1
$$

If the cost function in (13) is maximized, the inequality constraint in (13) is satisfied, which means that, while maximizing the cost function in (13), the equality constraint $\mathbf{w}^{H} \mathbf{c}_{1}-\delta\|\mathbf{w}\|=\kappa$ is satisfied, where $\kappa \geq 1$. Then, an extra constraint $\operatorname{Im}\left\{\mathbf{w}^{H} \mathbf{c}_{1}\right\}$ can be omitted because the equality constraint $\mathbf{w}^{H} \mathbf{c}_{1}-\delta\|\mathbf{w}\|=\kappa$ guarantees that the value of $\mathbf{w}^{H} \mathbf{c}_{1}$ is real-valued and positive [50]. Using the equality constraint, the weight vector $\mathbf{w}$ is obtained by rewriting the convex optimization problem of (13) as

$$
\underset{\mathbf{w}}{\operatorname{maximize}} E\left\{G\left(\left|\mathbf{w}^{H} \hat{\mathbf{y}}\right|^{2}\right)\right\} \text { subject to } \frac{1}{\delta^{2}}\left|\mathbf{w}^{H} \mathbf{c}_{1}-\kappa\right|^{2}=\mathbf{w}^{H} \mathbf{w} \text {. }
$$

This solves the optimization problem of worst-case performance by maximizing the approximate negentropy subject to the SOCP constraint. The constrained optimization problem in (14) will be derived by the quasi-Newton iteration. Newton's method is based on the Lagrangian function

$$
J(\mathbf{w})=E\left\{G\left(\left|\mathbf{w}^{H} \hat{\mathbf{y}}\right|^{2}\right)\right\}-\mu_{0}\left(\frac{1}{\delta^{2}}\left|\mathbf{w}^{H} \mathbf{c}_{1}-\kappa\right|^{2}-\mathbf{w}^{H} \mathbf{w}\right),
$$

where $\mu_{0}$ is a Lagrange multiplier. In order to avoid the influence of correlated noise subspace when decorrelating mixed-up signals, the pre-whitening step is omitted. Finally, the desired weight vector with unit variance constraint in the proposed method is as follows (details are presented in Appendix 1):

Step 1 . After centering $\hat{\mathbf{y}}$, take a small initial vector $\mathbf{w}(0)=0.01 \mathbf{c}_{1}$. Let iteration number $p=0$.

Step 2. Update

$$
\begin{aligned}
& \hat{\mathbf{w}}= \mathbf{R}_{\hat{\mathbf{y}}}^{-1} E\left\{\hat{\mathbf{y}}\left(\mathbf{w}^{H}(p) \hat{\mathbf{y}}\right)^{*} g\left(\left|\mathbf{w}^{H}(p) \hat{\mathbf{y}}\right|^{2}\right)\right\} \\
&-E\left\{g\left(\left|\mathbf{w}^{H}(p) \hat{\mathbf{y}}\right|^{2}\right)+\left|\mathbf{w}^{H}(p) \hat{\mathbf{y}}\right|^{2} g^{\prime}\left(\left|\mathbf{w}^{H}(p) \hat{\mathbf{y}}\right|^{2}\right)\right\} \mathbf{w}(p) \\
&+\mu \cdot \mathbf{R}_{\mathbf{y}}^{-1} \mathbf{c}_{1}, \\
& \mathbf{w}(p+1) \leftarrow \hat{\mathbf{w}} / \sqrt{\hat{\mathbf{w}}^{H} \mathbf{R}_{\hat{\mathbf{y}}} \hat{\mathbf{w}}}
\end{aligned}
$$

where $\mathbf{R}_{\hat{\mathbf{y}}}=E\left\{\hat{\mathbf{y}} \hat{\mathbf{y}}^{H}\right\}$ is the data correlation matrix and $g$ and $g^{\prime}$ denote the derivatives of $G$ and $g$, respectively.

Step 3. Check the convergence of $\mathbf{w}(p)$. If the error measure is $\sum_{m=1}^{M}\left|w_{m}(p+1)-w_{m}(p)\right|>\varepsilon$, where $\varepsilon$ is the terminating error value, let $p=p+1$ and go back to step 2. Otherwise, output the vector $\mathbf{w}(p)$. 
According to classical FastICA [36], the step that projects $\hat{\mathbf{w}}$ through $\mathbf{w}(p+1) \leftarrow \hat{\mathbf{w}} / \sqrt{\hat{\mathbf{w}}^{H} \mathbf{R}_{\hat{\mathbf{y}}} \hat{\mathbf{w}}}$ could enforce the unit variance constraint $E\left\{\left|\mathbf{w}^{H} \hat{\mathbf{y}}\right|^{2}\right\}=1$ after each step.

For coherent detection, a new modification of the classical FastICA has been proposed that omits the complex pre-whitening step and directly incorporates the SOCP constraint onto the correct solution. Of course, the desired spreading code is still necessary as a starting point. In the coherent MC-CDMA system, the proposed coherent SOCP-ICA detection is developed to combat small-to-medium, norm-bounded CEM under colored noise. It is worth noting that the proposed coherent detector can be implemented by choosing a proper value for $\mu$ without estimating $\delta$. Due to the algorithmic parameter $\mu$ in (16) (including to the Lagrange multiplier $\mu_{0}$, the parameter $\kappa$, and the unknown CEM $\delta$ ), the proposed SOCP-ICA possesses the capability of self-adjusting computation against CEM. Self-adjusting computation refers to a model of computing where computations can automatically respond to changes in varying $\mathrm{CEM} \delta$, which means that parameter $\mu$ can be kept a proper constant under varying circumstances by using self-adjusted $\mu_{0}$ and $\kappa$ for ICA iterations. The proposed algorithm is nonsensitive to small-to-medium CEM.

\subsection{Differential SOCP-ICA detection}

For a noncoherent receiver, DPSK is usually used to avoid channel estimation and tracking over a timevarying fading scenario [8]. When using the DPSK format, information is encoded in the phase difference between two successive symbol transmissions and the sequence of the transmitted data symbols for user $k$ is given by $b_{k}(i)=a_{k}(i) b_{k}(i-1)$, where $a_{k}(i)$ is the unmodulated data. In order to decorrelate observed mixtures, after centering $\mathbf{y}$, the observed data are first projected on the signal eigensubspace for the pre-processing step. To accomplish this, the transformation is given by

$$
\tilde{\mathbf{y}}=\mathbf{E}_{\mathrm{s}} \mathbf{E}_{\mathrm{s}}^{H} \mathbf{y}
$$

where $\mathbf{E}_{\mathrm{s}}$ is the signal subspace and corresponds to the principle eigenvectors of the data correlation matrix $\mathbf{R}_{\mathbf{y}}=E\left\{\mathbf{y y}^{{ }^{H}}\right\}$.

In contrast to the small-to-medium, norm-bounded CEM in (11), the formulation will be extended to a large-scale CEM over time-varying channels, which means that a differential SOCP-ICA criterion will not require the channel-state information of the desired user. The proposed differential detection maximizing the approximate negentropy that is subject to worst-case performance is still reasonably applied to the largely norm-bounded mismatch $\Delta \mathbf{e}$ and can be described in the following form:

$$
\underset{\mathbf{w}}{\operatorname{maximize}} E\left\{G\left(\left|\mathbf{w}^{H} \tilde{\mathbf{y}}\right|^{2}\right)\right\}, \quad \text { subject to } \min _{\|\Delta \mathbf{e}\| \leq \delta}\left|\mathbf{w}^{H} \hat{\mathbf{c}}_{1}\right| \geq 1 \text {. }
$$

Note that the bounded region $\delta$ of the differential scheme in (18) is different from the coherent in (11). The formulation will then be developed to overcome Doppler spread in the noncoherent MC-CDMA system under colored noise. Without channel estimation, the norm of $\Delta \mathbf{e}$ would be bounded by a large value $\delta$. The solution to (18) can then be found by maximizing the function

$$
J(\mathbf{w})=E\left\{G\left(\left|\mathbf{w}^{H} \tilde{\mathbf{y}}\right|^{2}\right)\right\}-\mu_{0}\left(\frac{1}{\delta^{2}}\left|\mathbf{w}^{H} \mathbf{c}_{1}-\kappa\right|^{2}-\mathbf{w}^{H} \mathbf{w}\right) .
$$

Next, the differential SOCP-ICA is performed as follows (details are similar to what is shown in Appendix 1):

Step 1. After centering $\mathbf{y}$ and projecting on the signal subspace, take a small initial vector $\mathbf{w}(0)=0.01 \mathbf{c}_{1}$. Let iteration number $p=0$.

Step 2. Update

$$
\begin{aligned}
& \tilde{\mathbf{w}}= \mathbf{R}_{\tilde{\mathbf{y}}}^{-1} E\left\{\tilde{\mathbf{y}}\left(\mathbf{w}^{H}(p) \tilde{\mathbf{y}}\right)^{*} g\left(\left|\mathbf{w}^{H}(p) \tilde{\mathbf{y}}\right|^{2}\right)\right\} \\
&-E\left\{g\left(\left|\mathbf{w}^{H}(p) \tilde{\mathbf{y}}\right|^{2}\right)+\left|\mathbf{w}^{H}(p) \tilde{\mathbf{y}}\right|^{2} g^{\prime}\left(\left|\mathbf{w}^{H}(p) \tilde{\mathbf{y}}\right|^{2}\right)\right\} \mathbf{w}(p) \\
&+\mu \cdot \mathbf{R}_{\tilde{\mathbf{y}}}^{-1} \mathbf{c}_{1}, \\
& \mathbf{w}(p+1) \leftarrow \tilde{\mathbf{w}} / \sqrt{\tilde{\mathbf{w}}^{H} \mathbf{R}_{\tilde{\mathbf{y}}} \tilde{\mathbf{w}}},
\end{aligned}
$$

where $\mathbf{R}_{\tilde{\mathbf{y}}}=E\left\{\tilde{\mathbf{y}} \tilde{\mathbf{y}}^{H}\right\}$ is the data correlation matrix.

Step 3. Check the convergence of $\mathbf{w}(p)$. If the error measure is $\sum_{m=1}^{M}\left|w_{m}(p+1)-w_{m}(p)\right|>\varepsilon$, where $\varepsilon$ is the terminating error value, let $p=p+1$ and go back to step 2 . Otherwise, output the vector $\mathbf{w}(p)$.

Finally, the decoding rule without training sequence for the desired user is given as $\hat{a}_{1}(i)=\operatorname{sign}\left(\operatorname{Re}\left\{\left(\mathbf{w}^{H} \mathbf{y}(i)\right)\right.\right.$ $\left.\left.\left(\mathbf{w}^{H} \mathbf{y}(i-1)\right)^{*}\right\}\right)$.

\section{Comparison of computational complexity}

The computational complexities of both the proposed coherent detector in (16) (including projection) and the differential SOCP-ICA detector in (20) (including preprocessing steps) are about $O\left(M^{2} B\right)$ in total (details are presented in Appendix 2).

The computational complexity of the proposed detectors will then be compared with the CMOE detector [15], the subspace-based MMSE detector [23], the robust VDL detector based on bounding probability [28], and the MMSEICA detector [39]. The evaluation of the CMOE detector including autocorrelation matrix $\mathbf{R}_{\hat{\mathbf{y}}}$ and its inverse operation $\mathbf{R}_{\hat{\mathbf{y}}}^{-1}$ has the complexity of order $O\left(M^{2} B\right)+O\left(M^{2}\right)=$ 
$O\left(M^{2} B\right)$. In the subspace-based MMSE detector, the EVD of the autocorrelation matrix has the complexity of $O\left(M^{2} B\right)+O\left(M^{2} K\right)$, and the complexity of the projection of the desired signature waveform onto signal subspace is $O\left(M^{2}\right)$. Thus, the final complexity of the subspace-based MMSE is of order $O\left(M^{2} B\right)$. The computation of the VDL detector has the complexity of $O\left(M^{2} B\right)$. Besides, in the MMSE-ICA, the computational complexities of autocorrelation matrix, prior subspace estimation, pre-whitening of the received data, and each unit-gainbased ICA iteration are $O\left(M^{2} B\right), O\left(M^{2} K\right), O(M K)$, and $O(K B)$, respectively. Thus, the final complexity of the MMSE-ICA is of order $O\left(M^{2} B\right)$.

\section{Simulation results}

Consider a synchronous multiuser MC-CDMA system under colored noise environment, using the following fixed parameters: the central frequency $f_{\mathrm{c}}=5.2 \mathrm{GHz}$, number of subcarrier $M=64$, sampling rate $20 \mathrm{MHz}$, Doppler spread equaling $20 \mathrm{~Hz}$. The length of the adopted WalshHadamard code is equal to 64 chips. Thus, the MCCDMA system can support the maximum number of active users $K=64$. At the desired receiver end, an unknown Rayleigh multipath fading channel model is adopted where the channel consists of $L_{1}=4$ multipath with gains $\beta_{1}(l)$ being independent, identically distributed complex Gaussian random variables with zero mean, identical variance $1 /$ $L_{1}$, and delay uniform over $\left[0,3 T_{\mathrm{c}}\right]$. Now, it is appropriately assumed that the small-to-medium CEM $\Delta \mathbf{e}^{\sim} N$ $\left(0, \sigma_{e}^{2}\right)$ happens at the receiver end. An additional $15 T_{\mathrm{c}}$ guard interval duration is used to provide protection from ISI due to channel multipath delay spread. The additive colored noise can be modeled by a moving average (MA) process [51] and generated by applying a white Gaussian noise sequence to a corresponding finite impulse response (FIR) filter with transfer function $H(z)=1-\alpha z^{-1}$ [52], where $\alpha$ is the correlation coefficient. In order to analyze the performance of coherent and differential detectors under the scenario of highly correlated noise, the correlated coefficient $\alpha$ is equal to 0.8 . The power of each interfering user is $3 \mathrm{~dB}$ more than that of the desired user at each trial.

For the proposed coherent SOCP-ICA in (16) and the differential SOCP-ICA in (20), the parameter values $\mu$ are equal to 0.91 and 10 , respectively. The terminating errors of both the iterative processes are set to $10^{-5}$ at each trial. For comparison purposes, the performance is also compared with the CMOE [15], the subspace-based MMSE [23], the robust VDL [28], and the MMSE-ICA [39] methods. The number of data symbols taken in the simulations is 1,000 data symbols at each trial. The number of Monte Carlo simulations is averaged over 5,000 independent trials.

First, Figure 1 shows the desired symbol error rate (SER) performance versus the received symbol size for each detector while the number of active users $K=15$, the desired user's SNR $=15 \mathrm{~dB}$, and CEM $\delta=0.1$. It can be found that when sample size is 600 data symbols, the proposed blind differential SOCP-ICA detector can achieve steady performance, and all the other coherent and differential detectors can achieve steady performance within 1,000 data symbols. The results show the speed of convergence of all the schemes and confirm that the 1,000-symbol is enough implementing for all detectors.

Then, consider the influence of the correlated coefficient $\alpha$ on each blind coherent detector with perfect channel estimation and blind DPSK detector. Figure 2

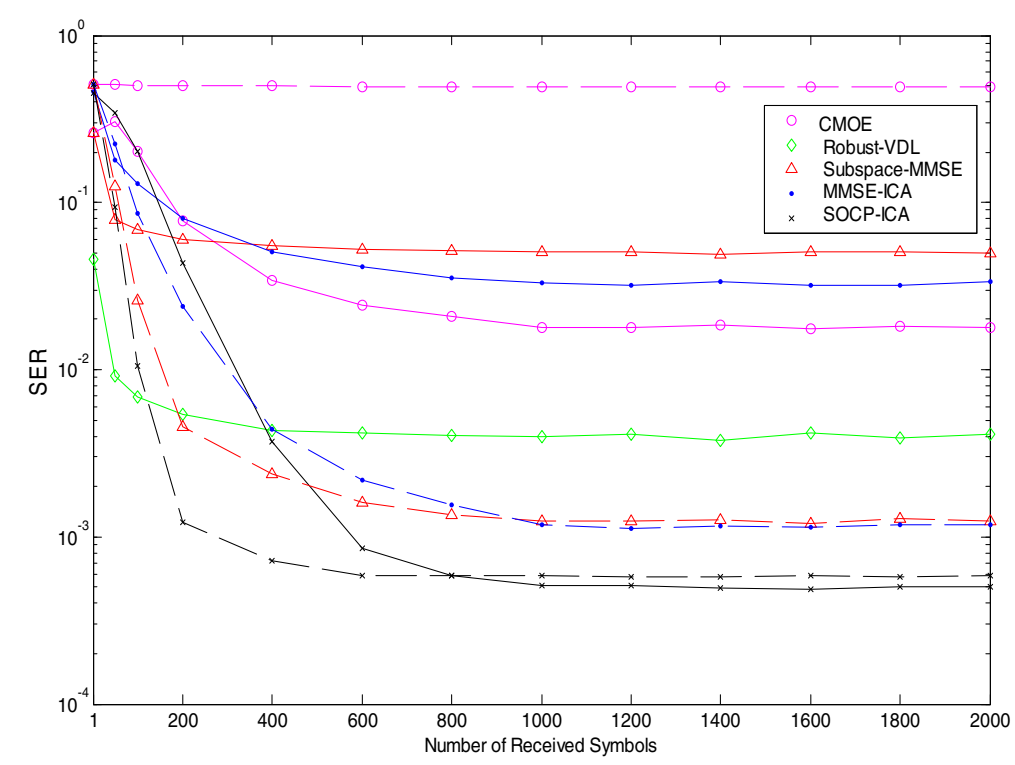

Figure 1 SER performance versus the received symbol size (-: coherent, - - -: differential). 


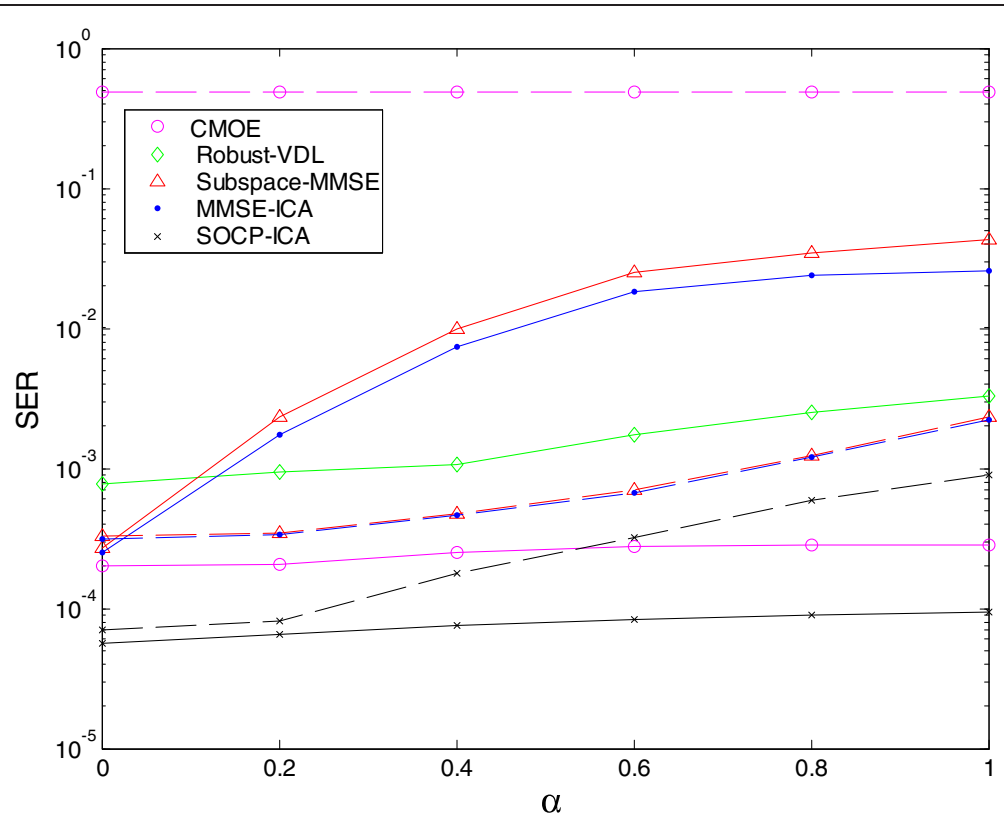

Figure 2 SER versus the correlated coefficient $\boldsymbol{a}$. (-: coherent, - - -: differential).

depicts the sensitivity in terms of the SER versus $\alpha$ while $K=15, \mathrm{SNR}=15 \mathrm{~dB}$, and $\delta=0$. In general, the performance of the coherent scheme is better than the differential under AWGN noise. The subspace-based MMSE and MMSE-ICA coherent detectors are much more sensitive to the correlated coefficient than the others since parts of the variation of the noise subspace diverge into the signal eigenvectors causing serious destruction to this orthogonality. The blind differential CMOE cannot capture the desired symbols and actually diverges after decoding. The proposed blind coherent SOCP-ICA detector in (16) omitting the complex pre-whitening step based on SOS eigenvector projection has the best performance; the proposed blind differential SOCP-ICA detector degrades with increasing the correlated coefficient $\alpha$ but is still better than the other blind differential ones. Clearly, HOS-based separation technique is less sensitive to colored noise compared to SOS-based EVD.

Figure 3 represents the sensitivity about the SER versus the CEM $\delta$ for $K=15$ and SNR $=15 \mathrm{~dB}$. For coherent

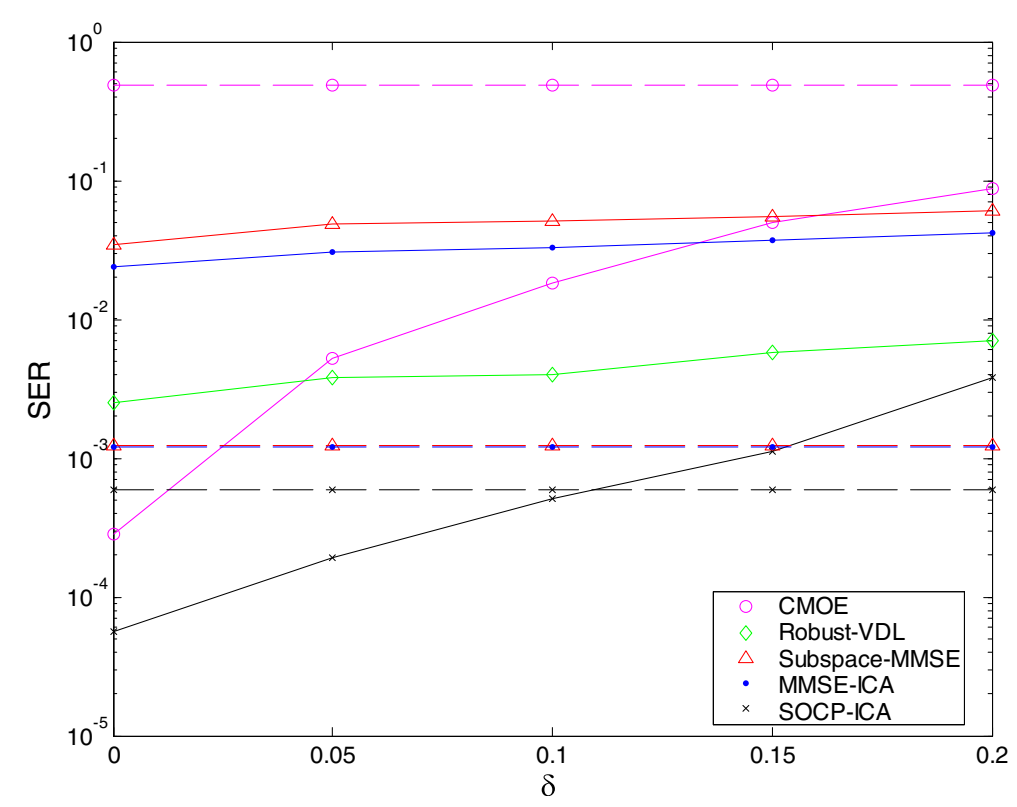

Figure 3 SER versus the CEM $\delta$. (-: coherent, - - -: differential). 


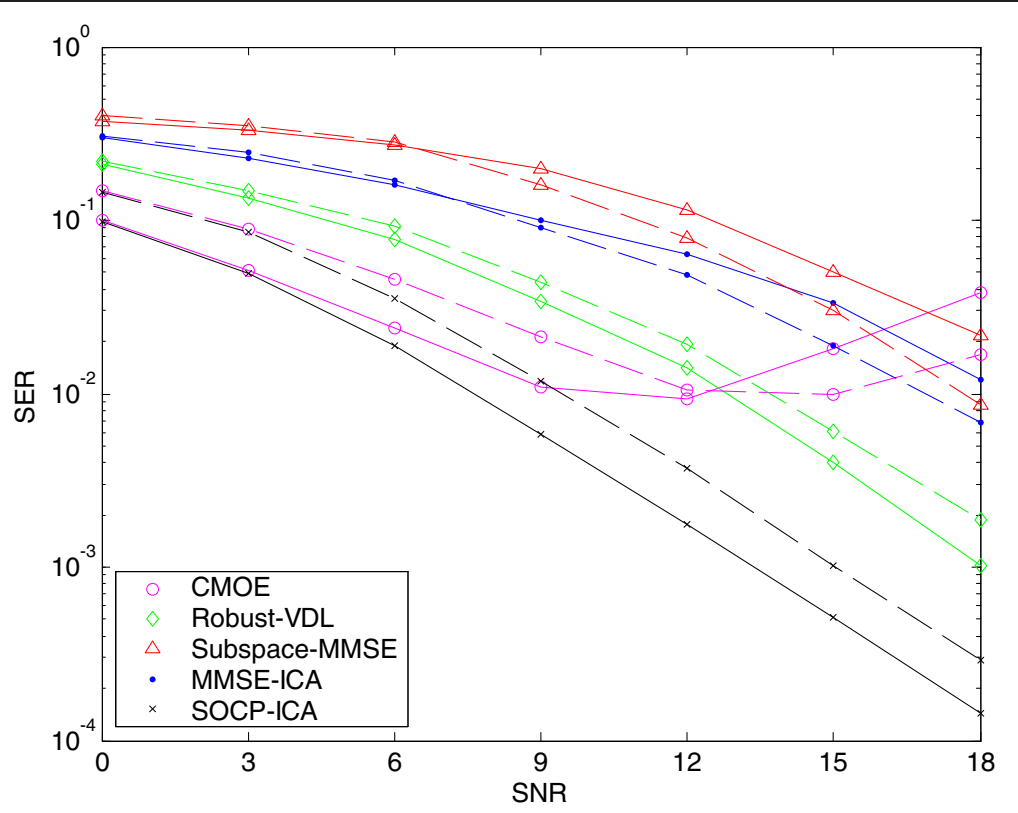

Figure 4 SER of the coherent schemes versus the desired user's input SNR. $(-: K=15,--; K=30)$.

schemes, the coherent CMOE is sensitive to the estimation mismatch which can lead to serious errors. The VDL detector based on the bounded condition provides a design trade-off between sensitivity and interference suppression. The proposed coherent SOCP-ICA detector which is a HOS-based source separation technique subject to SOCP constraint can provide robustness in the presence of small-to-medium channel mismatch and heavily correlated coefficients and substantially improves the system performance. The differential modulation schemes do not need channel estimation and compensation and are independent with $\delta$. Once $\delta>0.11$, the proposed blind differential SOCP-ICA detector process is much more robust than the coherent SOCP-ICA.

Figure 4 shows the SER of the coherent schemes for $K=15$ and $K=30$ as a function of the desired user's input SNR while CEM $\delta=0.1$. It is noticed that the performance of the coherent $\mathrm{CMOE}$ detector could not get better while SNR is increasing. The CMOE detector based on the optimal solution enforces the response of the interest signal to be of unity and minimizes the total variance of the detector output. While CEM $\delta$ exists in the constraint of the CMOE detector, the response of the interest signal is no longer constrained to be of unity. A part of the power of the interest signal will donate towards the interference and thus is seriously degraded by the CMOE method while minimizing the total variance of the detector output. This phenomenon is called signal cancellation or self-nulling. About the subspace-based MMSE and MMSE-ICA coherent detectors, it is seen that the performance for $K=30$ becomes better than that for $K=15$ at a larger SNR region. This performance improvement can be explained by the fact that the subspace-based methods are less sensitive to the heavily correlated coefficient by reason of the influence of noise subspace on higher-power signal eigenvectors becoming weaker while increasing more users. The performance of the proposed coherent SOCP-ICA detector is more robust than that of the other coherent ones. Figure 5 shows the SER of the differential schemes for $K=15$ and $K=30$ as a function of the desired user's input SNR. From Figures 4 and 5, it can be seen that the proposed SOCPICA detectors have better performance compared with the others. Figure 6 displays a comparative analysis of the differential schemes and the coherent schemes with perfect channel-state information for $K=15$. It is shown that there is an approximately 3- to 4- $\mathrm{dB}$ gap between the differential and the coherent SOCP-ICA with higher SNR.

The near-far effect is exhibited in Figure 7 that the power of each interfering user is more than the desired user's input power for $K=15, \mathrm{SNR}=15 \mathrm{~dB}$, and CEM $\delta=0.1$. The near-far resistance of the proposed SOCP-ICA detectors have better performance than the other ones, and the differential SOCP-ICA is especially the best while each interference-to-noise ratio (INR) is greater than $8 \mathrm{~dB}$.

Figure 8 shows the error measure versus the average number of iterations $p$ for $K=15$, SNR $=15 \mathrm{~dB}$, and CEM $\delta=0.1$. Here, coherent and differential SOCP-ICA detectors with four different numbers of users, $K$, are considered: $1,15,30$, and 64 . In order to attain the terminating error value $10^{-5}$, it can be observed that the larger $K$ is, the more the iteration numbers are. In general, the differential SOCP-ICA converges much faster than the coherent SOCP-ICA. 


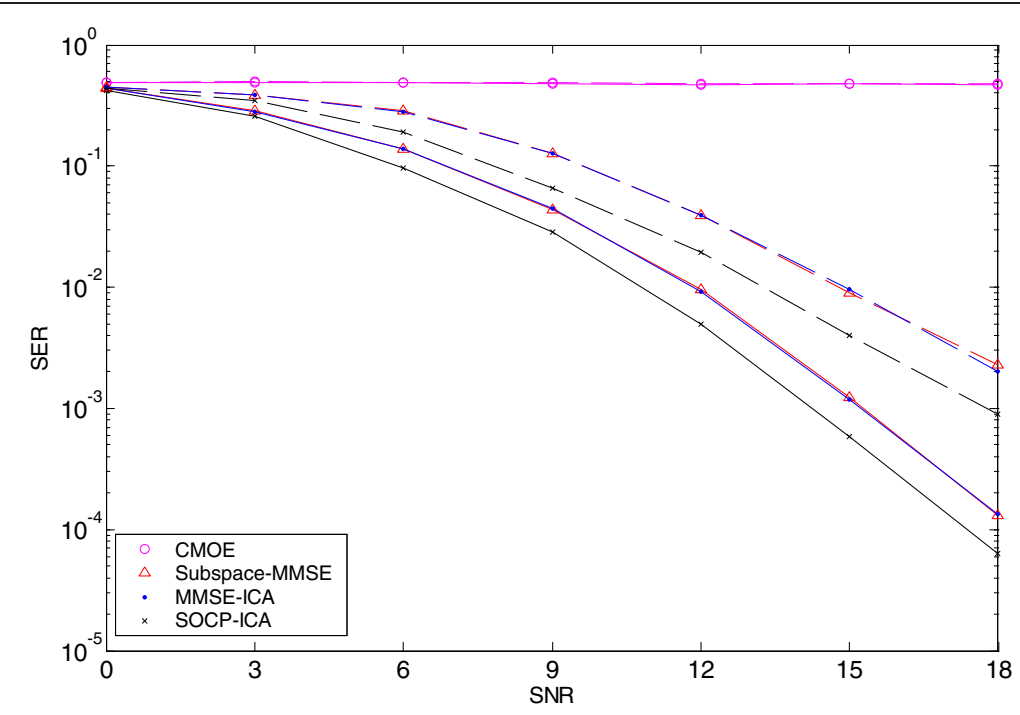

Figure 5 SER of the differential schemes versus the desired user's input SNR. $(-: K=15,--: K=30)$.

The section will analyze the influence of time-varying fading channels. Figure 9 illustrates the performance of the coherent and differential schemes with a high Doppler spread of $200 \mathrm{~Hz}$ for $K=15$ and CEM $\delta=0.1$. Then, Figure 10 illustrates the performance of the differential schemes over fast fading channels with a Doppler spread of $2 \mathrm{MHz}$. Here, 1,000 data symbols are used throughout the system at each trial. The notable feature of Figures 9 and 10 is that all the coherent schemes fail in $200 \mathrm{~Hz}$ and the significant performance of the differential SOCP-ICA detector is robust to rapid channel time variations and outperforms the other differential ones. Figure 11 compares the performance of the proposed differential SOCP-ICA employing symbol sizes 1,000 and 2,000 over time-varying fading channels with Doppler spreads of 200 and $2 \mathrm{MHz}$. The results show that there is an obvious difference in performance for different Doppler spread values under the desired user's input SNR $>18 \mathrm{~dB}$ and that both different symbol sizes have almost the same performance over the same fading scenario.

Figure 12 shows a comparative analysis between two different sets of delay taps $\left[0, T_{\mathrm{c}}, 2 T_{\mathrm{c}}, 3 T_{\mathrm{c}}\right]$ and $\left[0,4 T_{\mathrm{c}}\right.$, $8 T_{\mathrm{c}}, 12 T_{\mathrm{c}}$ ] with equal Doppler spread of $20 \mathrm{~Hz}$ at the four diversity branches for $K=15$ and CEM $\delta=0.1$. Due to slightly time-varying fading channels, the coherent detectors degrade with increasing the delay taps.

In summary, the outcomes of these experiments have illustrated that the proposed blind coherent SOCP-ICA

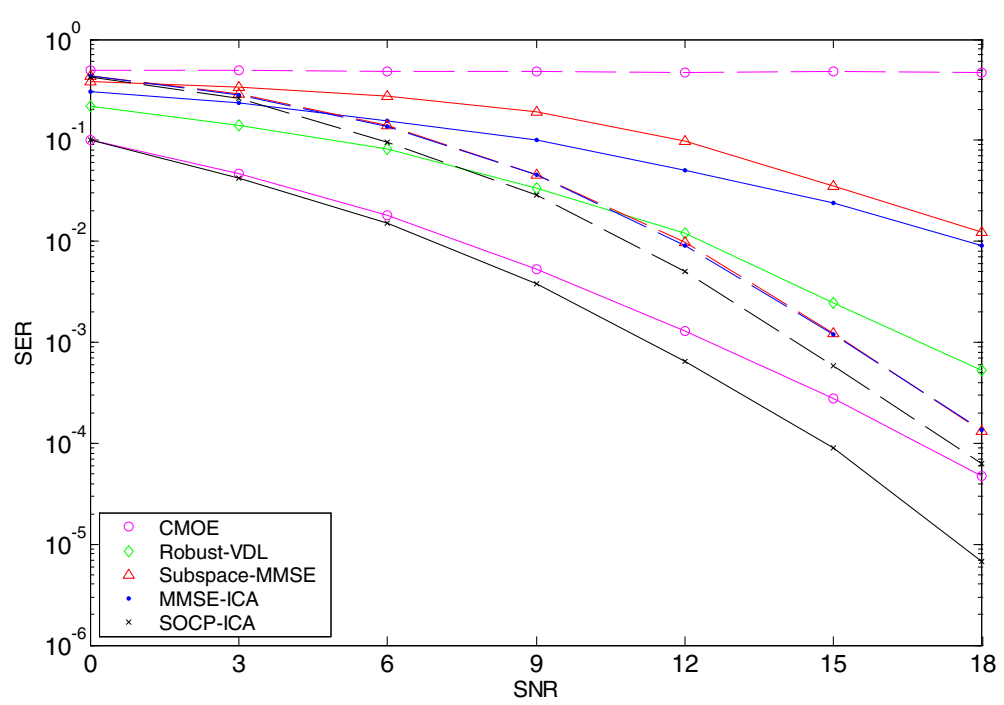

Figure 6 SER of the differential and perfect coherent schemes versus the desired user's input SNR. (—: coherent, - - : differential). 


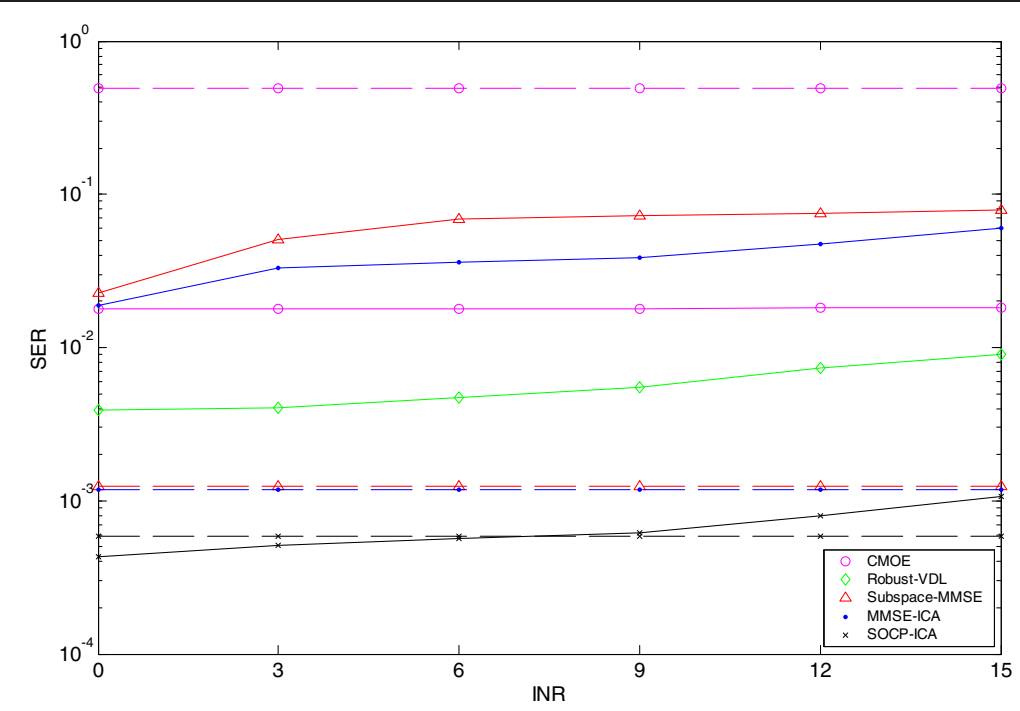

Figure 7 SER versus the strong INR of each interfering user. (-: coherent, - - -: differential).

detector can provide robustness against small-to-medium CEM and the blind differential SOCP-ICA detector can successfully overcome Doppler spread under colored noise environment.

\section{Conclusions}

In this paper, the proposed ICA based on approximate negentropy maximization subject to SOCP constraint has been applied to blind multiuser detection for a synchronous MC-CDMA system. The proposed blind coherent SOCP-ICA detector can provide robustness against smallto-medium CEM under colored noise to achieve better performance than the other coherent ones. Besides, the proposed blind differential SOCP-ICA can successfully overcome Doppler spread over heavily time-varying fading channels with colored noise. Simulation results have demonstrated that the new blind coherent and differential SOCP-ICA detectors substantially improve the system performance.

\section{Appendix 1}

This Appendix provides the derivation of (16). According to a new cost function in (15), its Jacobian vector is

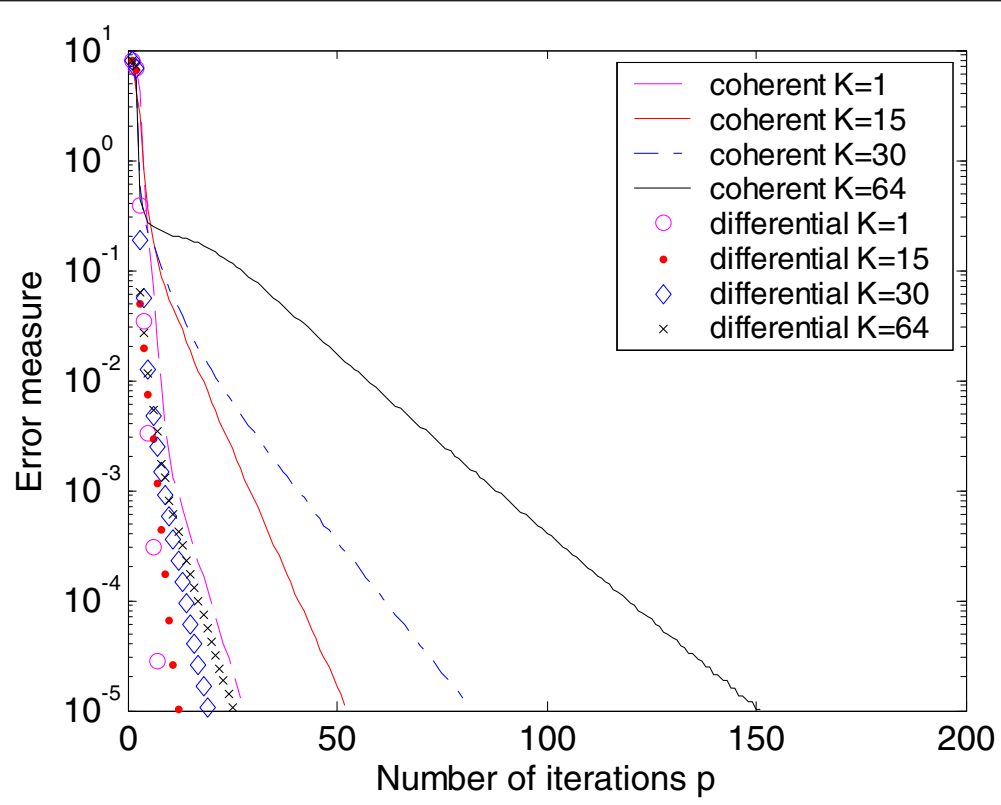

Figure 8 Error measure versus the average number of iterations $p$ for different number of users $K$. 


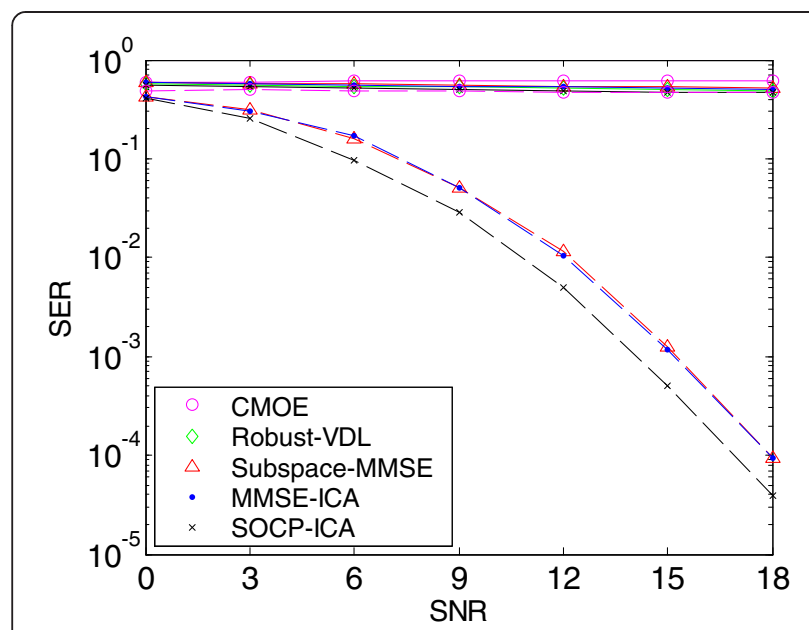

Figure 9 SER versus the desired user's input SNR for Doppler spread of $200 \mathrm{~Hz}$. (-: coherent, - - : differential).

$$
\nabla J(\mathbf{w})=2 E\left\{\hat{\mathbf{y}}\left(\mathbf{w}^{H} \hat{\mathbf{y}}\right)^{*} g\left(\left|\mathbf{w}^{H} \hat{\mathbf{y}}\right|^{2}\right)\right\}-\mu_{0}\left[\frac{1}{\delta^{2}}\left(\mathbf{c}_{1} \mathbf{c}_{1}^{T} \mathbf{w}-\kappa \mathbf{c}_{1}\right)-\mathbf{w}\right]
$$

Using the Newton iteration technique solving (14), the optimum of the cost function $E\left\{G\left(\left|\mathbf{w}^{H} \hat{\mathbf{y}}\right|^{2}\right)\right\}$ subject to the constraint $\left(1 / \delta^{2}\right)\left|\mathbf{w}^{H} \mathbf{c}_{1}-\kappa\right|^{2}=\mathbf{w}^{H} \mathbf{w}$ is obtained at points where $\nabla J(\mathbf{w})=0$. Then, the Jacobian matrix of $\nabla E\left\{G\left(\left|\mathbf{w}^{H} \hat{\mathbf{y}}\right|^{2}\right)\right\}$ can be approximated as

$$
\begin{aligned}
& \nabla^{2} E\left\{G\left(\left|\mathbf{w}^{H} \hat{\mathbf{y}}\right|^{2}\right)\right\}=2 E\left\{\hat{\mathbf{y}} \hat{\mathbf{y}}^{H} g\left(\left|\mathbf{w}^{H} \hat{\mathbf{y}}\right|^{2}\right)+\hat{\mathbf{y}} \hat{\mathbf{y}}^{H}\left|\mathbf{w}^{H} \hat{\mathbf{y}}\right|^{2} g^{\prime}\left(\left|\mathbf{w}^{H} \hat{\mathbf{y}}\right|^{2}\right)\right\} \\
& \approx 2 E\left\{\hat{\mathbf{y}} \hat{\mathbf{y}}^{H}\right\} E\left\{g\left(\left|\mathbf{w}^{H} \hat{\mathbf{y}}\right|^{2}\right)+\left|\mathbf{w}^{H} \hat{\mathbf{y}}\right|^{2} g^{\prime}\left(\left|\mathbf{w}^{H} \hat{\mathbf{y}}\right|^{2}\right)\right\}
\end{aligned}
$$

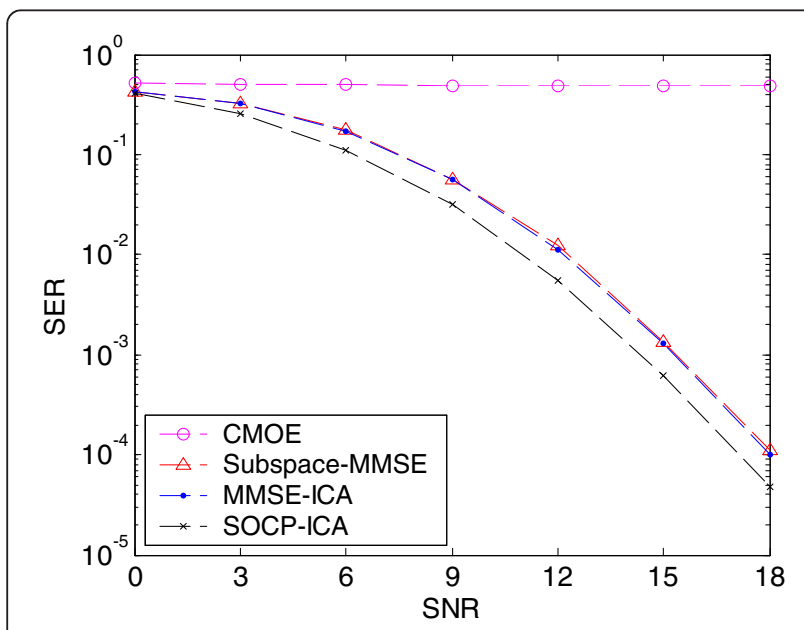

Figure 10 SER of the differential schemes versus the desired user's input SNR for Doppler spread of $2 \mathrm{MHz}$.

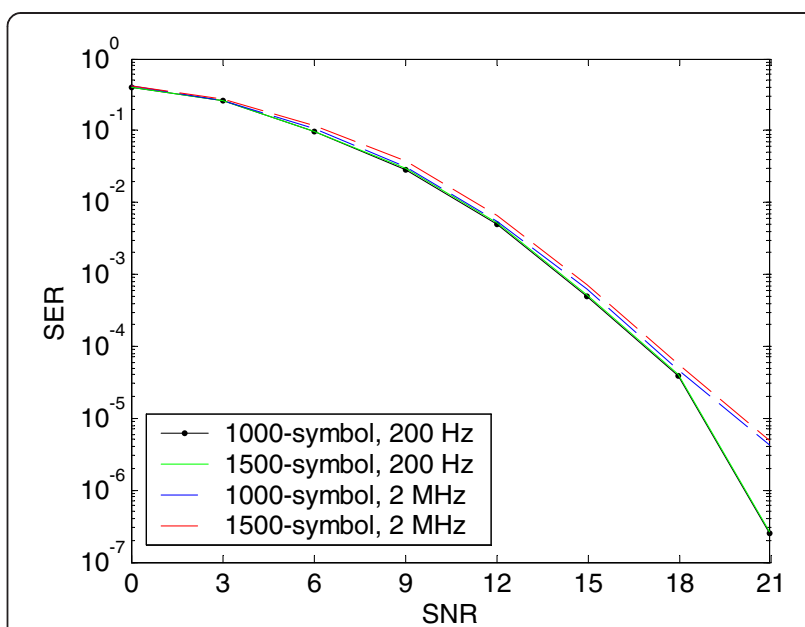

Figure 11 SER versus the desired user's input SNR for different sample sizes and Doppler spreads.

where the approximation is done by separating the expectations. The Jacobian of $\left(1 / \delta^{2}\right)\left(\mathbf{c}_{1} \mathbf{c}_{1}^{T} \mathbf{w}-K \mathbf{c}_{1}\right)-\mathbf{w}$ is $\left(1 / \delta^{2}\right) \mathbf{c}_{1} \mathbf{c}_{1}^{T}-\mathbf{I}$, where $\mathbf{I}$ is the identity matrix. Therefore, the total approximate Jacobian of (21) is

$$
\begin{aligned}
\nabla(\nabla J(\mathbf{w}))= & 2 E\left\{\hat{\mathbf{y}} \hat{\mathbf{y}}^{H}\right\} E\left\{g\left(\left|\mathbf{w}^{H} \hat{\mathbf{y}}\right|^{2}\right)+\left|\mathbf{w}^{H} \hat{\mathbf{y}}\right|^{2} g^{\prime}\left(\left|\mathbf{w}^{H} \hat{\mathbf{y}}\right|^{2}\right)\right\} \\
& -\mu_{0}\left[\left(1 / \delta^{2}\right) \mathbf{c}_{1} \mathbf{c}_{1}^{T}-\mathbf{I}\right] .
\end{aligned}
$$

To utilize the approximate Newton iteration, the weightupdating algorithm is given by

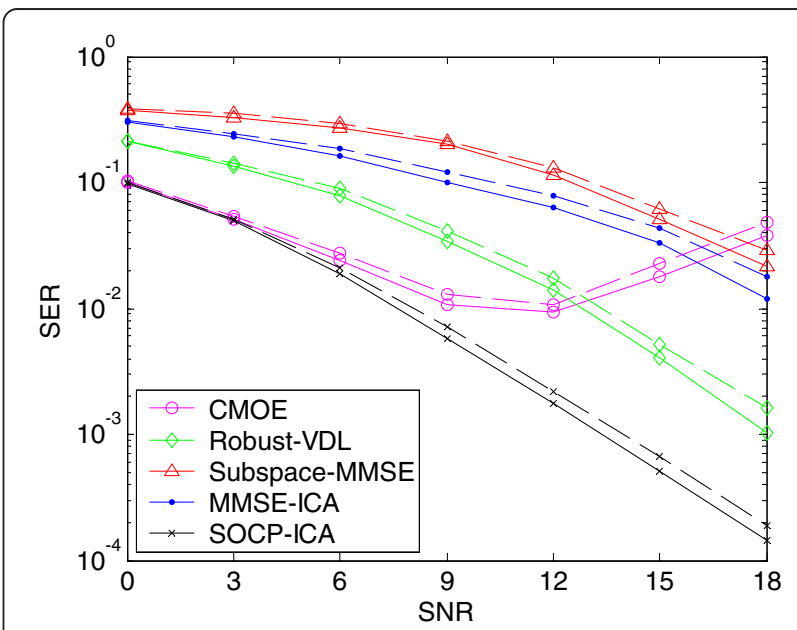

Figure 12 SER of the coherent schemes versus the desired user's input SNR for two sets of delay taps. (-: $\left[0, T_{c}, 2 T_{c}, 3 T_{c}\right]$, - - : [0, 4T $\left.T_{c}, 8 T_{c}, 12 T_{c}\right]$ ). 


$$
\begin{aligned}
& \mathbf{w}=\mathbf{w}-\frac{\nabla J(\mathbf{w})}{\nabla(\nabla J(\mathbf{w}(p)))} \\
& =\mathbf{w}-\frac{E\left\{\hat{\mathbf{y}}\left(\mathbf{w}^{H} \hat{\mathbf{y}}\right)^{*} g\left(\left|\mathbf{w}^{H} \hat{\mathbf{y}}\right|^{2}\right)\right\}-\left(\mu_{0} / 2\right)\left[1 / \delta^{2}\left(\mathbf{c}_{1} \mathbf{c}_{1}^{T} \mathbf{w}-\kappa \mathbf{c}_{1}\right)-\mathbf{w}\right]}{E\left\{\hat{\mathbf{y}} \hat{\mathbf{y}}^{H}\right\} E\left\{g\left(\left|\mathbf{w}^{H} \hat{\mathbf{y}}\right|^{2}\right)+\left|\mathbf{w}^{H} \hat{\mathbf{y}}\right|^{2} g^{\prime}\left(\left|\mathbf{w}^{H} \hat{\mathbf{y}}\right|^{2}\right)\right\}-\left(\mu_{0} / 2\right)\left[1 / \delta^{2}\left(\mathbf{c}_{1} \mathbf{c}_{1}^{T}\right)-\mathbf{I}\right]} .
\end{aligned}
$$

The algorithm can be further simplified by multiplying both sides of (24) by $-(1 / 2) \mathbf{R}_{\hat{y}}^{-1} \nabla(\nabla J(\mathbf{w}))$. While choosing a sufficiently small $\mu_{0}$, it can be easily shown that the left-hand side of the equality in (24) $\mathbf{w}$ multiplying $(1 / 2) \mathbf{R}_{\hat{\mathbf{y}}}^{-1} \nabla(\nabla J(\mathbf{w}))$ can be set as $\hat{\mathbf{w}}=\gamma \cdot \mathbf{w}$, where $\quad \gamma$ $=-E\left\{g\left(\left|\mathbf{w}^{H} \hat{\mathbf{y}}\right|^{2}\right)+\left|\mathbf{w}^{H} \hat{\mathbf{y}}\right|^{2} g^{\prime}\left(\left|\mathbf{w}^{H} \hat{\mathbf{y}}\right|^{2}\right)\right\} \in R$. Then, projecting $\hat{\mathbf{w}}$ through $\mathbf{w}(p+1) \leftarrow \hat{\mathbf{w}} / \sqrt{\hat{\mathbf{w}}^{H} \mathbf{R}_{\hat{\mathbf{y}}} \hat{\mathbf{w}}}$ could enforce the unit variance constraint $E\left\{\left|\mathbf{w}^{H} \hat{\mathbf{y}}\right|^{2}\right\}=1$ after each ICA iteration. The scalar factor $\gamma$ will not affect the convergence point. Finally, it can be explicitly simplified as

$$
\begin{aligned}
& \hat{\mathbf{w}}= \mathbf{R}_{\hat{\mathbf{y}}}^{-1} E\left\{\hat{\mathbf{y}}\left(\mathbf{w}^{H}(p) \hat{\mathbf{y}}\right)^{*} g\left(\left|\mathbf{w}^{H}(p) \hat{\mathbf{y}}\right|^{2}\right)\right\} \\
&-E\left\{g\left(\left|\mathbf{w}^{H}(p) \hat{\mathbf{y}}\right|^{2}\right)+\left|\mathbf{w}^{H}(p) \hat{\mathbf{y}}\right|^{2} g^{\prime}\left(\left|\mathbf{w}^{H}(p) \hat{\mathbf{y}}\right|^{2}\right)\right\} \mathbf{w}(p) \\
&+\mu \cdot \mathbf{R}_{\hat{\mathbf{y}}}^{-1} \mathbf{c}_{1}, \\
& \mathbf{w}(p+1) \leftarrow \hat{\mathbf{w}} / \sqrt{\hat{\mathbf{w}}^{H} \mathbf{R}_{\hat{\mathbf{y}}} \hat{\mathbf{w}}}
\end{aligned}
$$

where $\mu=\left(\mu_{0} \kappa / 2 \delta^{2}\right)$.

\section{Appendix 2}

The desired weight vector of the coherent SOCP-ICA detector can be obtained by calculating (16). It is assumed that the number of symbols $B$ is used for each batching processing to form the data matrix $\hat{\mathbf{Y}} \equiv[\hat{\mathbf{y}}(1), \hat{\mathbf{y}}(2), \cdots$, $\hat{\mathbf{y}}(B)]$. First, the computational complexity of computing the autocorrelation matrix $\mathbf{R}_{\hat{\mathbf{y}}}=E\left\{\hat{\mathbf{y}} \hat{\mathbf{y}}^{H}\right\}=(1 / B) \sum_{i=1}^{B} \hat{\mathbf{y}}$ $(i) \hat{\mathbf{y}}(i)^{H}$ needs $M^{2} B$ multiplications implementing and is thus of order $O\left(M^{2} B\right)$. The inverse autocorrelation matrix $\mathbf{R}_{\hat{\mathrm{y}}}^{-1}$ can be improved from $O\left(M^{3}\right)$ to $O\left(M^{2}\right)$ [53]. Then, the iteration step used by the formula in (16) is

$$
\hat{\mathbf{w}} \leftarrow \mathbf{R}_{\hat{\mathbf{y}}}^{-1}\left(\mathbf{h}(p)+\mu \mathbf{c}_{1}\right)-\chi(p) \mathbf{w}(p),
$$

where $\mathbf{h}(p)=(1 / B) \sum_{i=1}^{B} \mathbf{h}_{i}(p)$, the vector $\mathbf{h}_{i}(p)$ is the $i$ th vector of $\left\{\hat{\mathbf{Y}} \square \mathbf{h}^{H}(p)\right\}, \square$ is the Khatri-Rao product, and $\mathbf{h}(p) \equiv g\left(\left|\mathbf{w}^{H}(p) \hat{\mathbf{Y}}\right|^{2}\right) \odot\left(\mathbf{w}^{H}(p) \hat{\mathbf{Y}}\right)$. Here, $|\cdot|^{2}$ is taken elementwise and $\odot$ stands for elementwise multiplication. Furthermore, $X(p)=(1 / B) \sum_{i=1}^{B} X_{i}(p)$, the $X_{i}(p)$ is the $i$ th data of $\chi(p) \equiv g\left(\left|\mathbf{w}^{H}(p) \hat{\mathbf{Y}}\right|^{2}\right)+g^{\prime}\left(\left|\mathbf{w}^{H}(p) \hat{\mathbf{Y}}\right|^{2}\right) \odot\left|\mathbf{w}^{H}(p) \hat{\mathbf{Y}}\right|^{2}$. $\mathbf{w}^{H} \hat{\mathbf{Y}}$ is of order $O(M B) . \mathbf{h}(p)$ is of order $O(M B)+O(B)$, where the last term is due to the elementwise multiplications. The computational complexity of $\mathbf{R}_{\hat{\mathbf{y}}}^{-1}$ multiplying by an $M \times 1$ vector is of order $O\left(M^{2}\right)$. The $\chi(p)$ is of order $O$
$(M B)+O(B)$. Consequently, the computational complexity of the proposed coherent detector in (16) including projection is of order about $O\left(M^{2} B\right)+O\left(M^{2}\right)+O(M B)+$ $O(B)=O\left(M^{2} B\right)$ (since $\left.B>M\right)$ in total.

The differential SOCP-ICA detector needs to additionally utilize the subspace-based pre-processing step in (17). The computational complexity of autocorrelation matrix $\mathbf{R}_{\tilde{y}}$ and its inverse operation is of order $O\left(M^{2} B\right)+$ $O\left(M^{2}\right)$. The computational complexity of the classical subspace approach is $O\left(M^{3}\right)$ for computing the EVD of a $M \times M$ dimensional matrix. If only $K$ eigenvectors are used, its computational complexity is reduced to $O\left(M^{2} K\right)$ by recursive algorithms [54]. The computational complexity of the pre-processing in (17) is of order $O\left(M^{2}\right)+O$ $(M B)$ for $B$ symbols. Therefore, the computational complexity of the differential SOCP-ICA detector in (20) including the pre-processing steps $O\left(M^{2} K\right)+O\left(M^{2}\right)+O$ $(M B)$ is of order about $O\left(M^{2} B\right)$ in total.

\section{Competing interests}

The authors declare that they have no competing interests.

Received: 25 December 2013 Accepted: 19 August 2014 Published: 8 October 2014

\section{References}

1. L Hanzo, B-J Choi, Near-instantaneously adaptive HSDPA-style OFDM versus MC-CDMA transceivers for WiFi, WiMAX, and next generation cellular systems. Proc. IEEE 95(12), 2368-2392 (2007)

2. THwang, $Y(G) L i, Y$ Yuan-Wu, Energy spreading transform for down-link MC-CDMA. IEEE Trans. Wireless Commun. 7(5), 1522-1526 (2008)

3. BA Al-fuhaidi, HEA Hassan, MM Salah, SS Alagooz, Parallel interference cancellation with different linear equalisation and rake receiver for the downlink MC-CDMA systems. IET Commun. 6(15), 2351-2360 (2012)

4. C-C Lin, W-C Chen, C-D Chung, Spectral sidelobe decaying property of Walsh-Hadamard code in MC-CDMA systems. IEEE Trans. Wireless Commun. 10(10), 3151-3156 (2011)

5. P Ting, C-K Wen, J-C Chen, J-T Chen, BER analysis of the optimum multiuser detection with channel mismatch in MC-CDMA systems. IEEE J. Select Area Commun. 24(6), 1221-1235 (2006)

6. JG Proakis, Digital Communications, 4th edn. (Prentice-Hall, Englewood Cliffs, 2001)

7. THimsoon, W Su, KJR Liu, Single-block differential transmit scheme for broadband wireless MIMO-OFDM systems. IEEE Trans. Signal Process. 54(9), 3305-3314 (2006)

8. SM Elnoubi, E Sourour, A Elshamly, Performance of multicarrier CDMA with DPSK modulation and differential detection in fading multipath channels. IEEE Trans. Veh. Technol. 51(3), 526-536 (2002)

9. M Morelli, M Moretti, Channel estimation in OFDM systems with unknown interference. IEEE Trans. Wireless Commun. 8(10), 5338-5347 (2009)

10. CR Berger, J Gomes, JMF Moura, Sea-trial results for cyclic-prefix OFDM with long symbol duration, in Proceedings of the MTS/IEEE OCEANS Conference (Santander, Spain, 2011)

11. CR Berger, W Chen, S Zhou, J Huang, A simple and effective noise whitening method for underwater acoustic orthogonal frequency division multiplexing. J. Acoust. Soc. Am. 127(4), 2358-2367 (2010)

12. Z Wang, S Zhou, GB Giannakis, CR Berger, J Huang, Frequency-domain oversampling for zero-padded OFDM in underwater acoustic communications. IEEE J. Ocean. Eng. 37(1), 14-24 (2012)

13. W Chen, J Huang, Z Wang, S Zhou, Blind channel shortening for zeropadded OFDM, in Proceedings of MTS/IEEE OCEANS Conference 2010 (Seattle, 2010), pp. 20-23

14. S Wu, Y Bar-Ness, A phase noise suppression algorithm for OFDM-based WLANs. IEEE Commun. Lett. 6(12), 535-537 (2002) 
15. M Honig, U Madhow, S Verdu, Blind adaptive multiuser detection. IEEE Trans. Inform. Theory 41(7), 944-960 (1995)

16. H Xiaodong Wang, $V$ Poor, Blind multiuser detection: a subspace approach. IEEE Trans. Inform. Theory 44(2), 677-690 (1998)

17. R Wang, H Li, T Li, Robust Multiuser Detection for Multicarrier CDMA Systems. Select Area Commun. 24(3), 673-683 (2006)

18. H Cheng, SC Chan, Blind linear MMSE receivers for MC-CDMA systems. IEEE Trans. Circuits Syst. 54(2), 367-376 (2007)

19. SA Vorobyov, Robust CDMA multiuser detectors: probability-constrained versus the worst-case-based design. IEEE Signal Process Lett. 15(7), 273-276 (2008)

20. L Hu, X Zhou, L Zhang, Blind multiuser detection based on Tikhonov regularization. IEEE Commun. Lett. 15(5), 482-483 (2011)

21. Z Xu, P Liu, X Wang, Blind multiuser detection: from MOE to subspace methods. IEEE Trans. Signal Process. 52(2), 510-524 (2004)

22. S-J Chern, P-S Chao, New Capon-like blind MIMO-CDMA receiver with LCCM-RLS algorithm for multipath time-varying channels, in Proc. of the 2008 IEEE International Symposium on Intelligent Signal Processing and Communications (Bangkok, 2008), pp. 102-105

23. P Xiao, R Liu, Multi-user detector for multi-carrier CDMA systems. Electronics Lett. 44(23), 1366-1368 (2008)

24. MC Dogan, JM Mendel, Applications of cumulants to array processing. I. Aperture extension and array calibration. IEEE Trans. Signal Process. 43(5), 1200-1216 (1995)

25. Y Ma, KH Li, AC Kot, G Ye, A blind code timing estimator and its implementation for DS-CDMA signals in unknown colored noise. IEEE Trans. Veh. Technol. 51(6), 1600-1607 (2002)

26. Z Tian, KL Bell, HL Van Trees, Robust constrained linear receivers for CDMA wireless systems. IEEE Trans. Signal Process. 49(7), 1510-1522 (2001)

27. A Elnashar, SM Elnoubi, HA El-Mikati, Further study on robust adaptive beamforming with optimum diagonal loading. IEEE Trans. Antennas Propag. 54(12), 3647-3658 (2006)

28. R Wang, H Li, T Li, Robust multiuser detection for multicarrier CDMA systems. IEEE J. Select Area Commun. 24(3), 673-683 (2006)

29. J-F Cardoso, Blind signal separation: statistical principles. Proc. IEEE 86(10), 2009-2025 (1998)

30. A Hyvärinen, E Oja, Independent component analysis: algorithms and applications. Neural Netw. 13(4-5), 411-430 (2000)

31. Y Washizawa, Y Yamashita, T Tanaka, A Cichocki, Blind extraction of global signal from multi-channel noisy observations. Trans. Neural Netw. 21(9), 1472-1481 (2010)

32. F Nesta, P Svaizer, M Omologo, Convolutive BSS of short mixtures by ICA recursively regularized across frequencies. Trans. Audio Speech Lang. Process 19(3), 624-639 (2011)

33. A Hyvärinen, Fast and robust fixed-point algorithms for independent component analysis. IEEE Trans. Neural Netw. 10(3), 626-634 (1999)

34. MA Vicente, PO Hoyer, A Hyvärinen, Equivalence of some common linear feature extraction techniques for appearance-based object recognition tasks. Trans. Pattern Anal Mach. Intell. 29(5), 896-900 (2007)

35. V Zarzoso, P Comon, R Phlypo, A contrast function for independent component analysis without permutation ambiguity. IEEE Trans. Neural Netw. 21(5), 863-868 (2010)

36. E Bingham, A Hyvärinen, A fast fixed-point algorithm for independent component analysis of complex-valued signals. Int. J. Neural Syst. 10(1), 1-8 (2000)

37. T Adah, H Li, M Novey, J-F Cardoso, Complex ICA using nonlinear functions. IEEE Trans. Signal Process. 56(9), 4536-4544 (2008)

38. M Novey, T Adalı, Complex ICA by negentropy maximization. IEEE Trans. Neural Netw. 19(4), 596-609 (2008)

39. T Ristaniemi, J Joutsensalo, Advanced ICA-based receivers for block fading DS-CDMA channels. Signal Process. 82(3), 417-431 (2002)

40. WY Leong, J Homer, Blind multiuser receiver for DS-CDMA wireless system. IEE Proc. Commun. 153(5), 733-739 (2006)

41. S-i Amari, Natural gradient works efficiently in learning. Neural Comput. 10, 251-276 (1997)

42. D Blanco, B Mulgrew, ICA in signals with multiplicative noise. IEEE Trans. Signal Process. 52(8), 2648-2657 (2005)

43. WY Leong, DP Mandic, Noisy component extraction. IEEE Trans. Circuit Syst. 57(3), 664-671 (2010)
44. J Igual, L Vergara, A Camacho, R Miralles, Independent component analysis with prior information about the mixing matrix. Neurocomputing 50, 419-438 (2003)

45. Z-L Sun, K-M Lam, Depth estimation of face images based on the constrained ICA model. Trans. Inform. Foren Sec. 6(2), 360-370 (2011)

46. Z-Q Luo, W Yu, An introduction to convex optimization for communications and signal processing. IEEE J. Select Area Commun. 24(8), 1426-1438 (2006)

47. S Cui, M Kisialiou, Z-Q Luo, Z Ding, Robust blind multiuser detection against signature waveform mismatch based on second-order cone programming. IEEE Trans. Wireless Commun. 4(4), 1286-1291 (2005)

48. A Papoulis, Probability, Random Variables, and Stochastic Processes, 4th edn. (McGraw-Hill, New York, 1991)

49. PA Regalia, E Kofidis, Monotonic convergence of fixed-point algorithms for ICA. IEEE Trans. Neural Netw. 14(4), 943-949 (2003)

50. SA Vorobyov, AB Gershman, Z-Q Luo, Robust adaptive beamforming using worst-case performance optimization: a solution to the signal mismatch problem. IEEE Trans. Signal Process. 51(2), 313-323 (2003)

51. C-Y Yang, B-S Chen, Adaptive channel-tracking method and equalization for MC-CDMA systems over rapidly fading channel under colored noise. EURASIP J. Adv. Signal Process, (2010). Article ID 871650

52. AJ Rojas, R Middleton, JS Freudenberg, Infimal feedback capacity for a class of additive coloured Gaussian noise channels, in Proceedings of the 17th IFAC World Congress, 2008

53. S Piotr, Dynamic transitive closure via dynamic matrix inverse: extended abstract, in Proceedings of the 45th Annual IEEE Symposium on Foundations of Computer Science, 2004, pp. 509-517

54. Y Kai-Bor, Recursive updating the eigenvalue decomposition of a covariance matrix. IEEE Trans. Signal Process. 39(5), 1136-1145 (1991)

doi:10.1186/1687-6180-2014-151

Cite this article as: Jen and Jou: Blind ICA detection based on secondorder cone programming for MC-CDMA systems. EURASIP Journal on Advances in Signal Processing 2014 2014:151.

\section{Submit your manuscript to a SpringerOpen ${ }^{\odot}$ journal and benefit from:}

- Convenient online submission

Rigorous peer review

- Immediate publication on acceptance

- Open access: articles freely available online

- High visibility within the field

- Retaining the copyright to your article

Submit your next manuscript at $>$ springeropen.com 